OPEN

SUBJECT AREAS:

GENE REGULATION

PEPTIDES

PHYSIOLOGY

PATHOLOGY

Received

1 March 2012

Accepted

14 May 2012

Published

30 May 2012

Correspondence and requests for materials should be addressed to K.S. (kristina. schoonjans@epfl.ch)

\section{TGR5 potentiates GLP-1 secretion in response to anionic exchange resins}

\author{
Taoufiq Harach', Thijs W. H. Pols', Mitsunori Nomura' ', Adriano Maida', Mitsuhiro Watanabe ${ }^{2}$ \\ Johan Auwerx' \& Kristina Schoonjans'
}

'Laboratory of Integrative and Systems Physiology (LISP), School of Life Sciences, Ecole Polytechnique Fédérale de Lausanne, $\mathrm{CH}$ 1015 Lausanne, Switzerland, ${ }^{2}$ Division of Molecular Metabolism and System Medicine, School of Medicine, Keio University, Tokyo, Japan.

Anionic exchange resins are bona fide cholesterol-lowering agents with glycemia lowering actions in diabetic patients. Potentiation of intestinal GLP-1 secretion has been proposed to contribute to the glycemia lowering effect of these non-systemic drugs. Here, we show that resin exposure enhances GLP-1 secretion and improves glycemic control in diet-induced animal models of "diabesity", effects which are critically dependent on TGR5, a G protein-coupled receptor that is activated by bile acids. We identified the colon as a major source of GLP-1 secretion after resin treatment. Furthermore, we demonstrate that the boost in GLP-1 release by resins is due to both enhanced TGR5-dependent production of the precursor transcript of GLP-1 as well as to the local enrichment of TGR5 agonists in the colon. Thus, TGR5 represents an essential component in the pathway mediating the enhanced GLP-1 release in response to anionic exchange resins.

ile acids (BAs) are molecules synthesized from cholesterol. They are composed of a sterol nucleus, a variable number of hydroxyl groups and a side chain with a carboxyl group that can be conjugated to taurine or glycine. The amphipathic nature of bile acids is essential for the emulsification and subsequent absorption of dietary lipids and fat-soluble vitamins. In addition to their established role in dietary lipid absorption, BAs have also emerged as important signaling molecules in various aspects of metabolic homeostasis ${ }^{1-4}$. Both nuclear and membrane receptors have been shown to mediate the transduction of bile acid signals into adaptive cellular responses. While the genomic effects of BAs are mainly governed by the nuclear receptor FXR, many of the rapid non-genomic effects have been attributed to activation of the membrane receptor TGR5.

TGR5 (Gpbar1, GPR131, M-BAR) is a G protein-coupled receptor, which upon binding by BAs stimulates downstream cAMP signaling pathways in a wide array of tissues and cell types ${ }^{5,6}$. Functional activation of TGR5 leads to the regulation of several physiological pathways that control BA and metabolic homeostasis. Actions of TGR5 relevant to BA homeostasis are evident from the observation that mice lacking TGR5 have a reduced BA pool size ${ }^{7,8}$ and from the finding that TGR5 activation promotes chloride and fluid secretion in gallbladder epithelium ${ }^{9}$. TGR5 activation also prevents body weight gain and hepatic steatosis during high fat feeding ${ }^{10,11}$ and inhibits inflammatory responses ${ }^{5,12-14}$. These properties of TGR5 suggest that its activation could beneficially impact on several features of the metabolic syndrome.

In the gut, BA dependent activation of TGR5 signaling improves glucose homeostasis by inducing glucagon-like peptide-1 (GLP-1) secretion ${ }^{11,15}$. The incretin, GLP-1, is a gut peptide derived from the precursor, preproglucagon, which is synthesized in the enteroendocrine L-cells of the intestinal epithelium ${ }^{16}$. Upon food ingestion, GLP-1 is released in the circulation causing a wide range of endocrine effects that directly or indirectly impact postprandial glucose homeostasis. The insulinotropic action of GLP-1 in pancreatic $\beta$-cells has been shown to mediate the glucose lowering effect of GLP-1, however, other GLP-1-dependent mechanisms, such as reduction of gastric emptying and food intake, also contribute to this effect ${ }^{17-20}$. Interestingly, recent evidence suggests that anionic exchange resins (AERs), which are non-absorbed polymers with bile acid sequestering properties, can improve glycemic control in type 2 diabetes ${ }^{21-25}$. Enhanced GLP-1 secretion has been proposed to account for the glucose lowering action of AERs ${ }^{26-29}$, but the molecular basis by which AERs increase GLP-1 secretion is still largely unexplored. GLP-1-producing enteroendocrine L-cells are highly enriched in colon, yet only minimal levels of BAs effectively reach the colon under physiological conditions. This is caused by the efficient re-uptake of BAs by the epithelial cells of the ileum, which is an integral part of the enterohepatic recycling of BAs. We therefore hypothesized that increasing the BA delivery to the colon with AERs may potentiate the TGR5-GLP-1 pathway. In the current study we demonstrate that 
TGR5 is essential for the AER-mediated increase in GLP-1 release and that the colon is a major site that accounts for this effect.

\section{Results}

AER-mediated stimulation of GLP-1 release is abolished in TGR5deficient mice. To test the hypothesis that TGR5 mediates the enhanced GLP-1 release induced by AERs, we treated TGR5 ${ }^{-1-}$ and $\mathrm{TGR}^{+/+}$mice with Colestilan ${ }^{30}$. Colestilan treatment, administered to the mice by food admix at a dose of $20 \mathrm{~g} / \mathrm{kg}$ of food $(2 \% \mathrm{w} / \mathrm{w})$, was initiated in mice 12 weeks after start of the high fat diet. After two weeks of treatment, several plasma and fecal lipid parameters were assessed. Administration of AERs decreased LDL-cholesterol in $\mathrm{TGR}^{+/+}$mice, whereas HDL- and total-cholesterol levels remained unaffected (Table 1). AER treatment also enriched fecal excretion of phospholipids, glycerol derivatives and non-esterified fatty acids to a similar extent in both genotypes (Table 1), except for the phospholipids, which were already high in the untreated TGR5 $5^{-I-}$ mice. As expected, AER treatment enriched fecal BA levels, although these levels were lower in both untreated and AER-treated TGR5 $5^{-1-}$ mice, which is consistent with the reduced BA pool size in TGR $5^{-1-}$ mice ${ }^{7,8}$.

In accordance with previous reports ${ }^{28,31,32}$, treatment with AERs robustly induced GLP-1 secretion in wildtype mice after a test meal (Fig. 1a). In $\mathrm{TGR}^{-1-}$ littermates, however, this response was severely blunted (Fig. 1b), indicating that TGR5 is an essential component in mediating the resin-induced increase in plasma GLP-1. We next investigated glucose homeostasis in obese and insulin resistant animals treated for two weeks with Colestilan. Test mealinduced insulin (Fig. 1c and 1d) and glucose (Fig. 1e and 1f) levels were significantly reduced in plasma of AER-treated TGR $5^{+/+}$mice whereas these effects were less pronounced in TGR5 $5^{-1-}$ mice. Resin treatment reduced body weight similarly across genotypes (Table 1), indicating that the differences in glucose handling were not the consequence of changes in body weight. These findings suggest that the TGR5-dependent improvement in glucose homeostasis in response to AERs may result from enhanced GLP-1 action.

TGR5 activation induces proglucagon gene expression and promoter activity in enteroendocrine cells. In order to gain insight into the mechanisms underlying the TGR5-dependent secretion of GLP-1 upon AERs, we first assessed expression of TGR5 in different sections of the intestine. We observed that AERs did not modulate TGR5 expression in the intestine, as TGR5 mRNA levels in Colestilan-treated $\mathrm{TGR}^{+/+}$intestinal sections were indistinguishable from those of untreated TGR $5^{+/+}$mice (Fig. 2a). Interestingly, in addition to enhanced GLP-1 secretion, mRNA levels of proglucagon, encoding the precursor of GLP-1, were also significantly increased in the colons of AER-treated $\mathrm{TGR} 5^{+/+}$mice relative to untreated $\mathrm{TGR}^{+/+}$mice. This increase in proglucagon mRNA was not observed in $\mathrm{TGR}^{-1-}$ mice (Fig. 2b). Although not statistically significant, a similar trend for proglucagon expression was found in the ileum (Fig. 2b). To investigate the mechanism by which TGR5 regulates proglucagon gene expression, TGR5 was silenced in cultured STC-1 and GLUTag mouse enteroendocrine cells using shRNA constructs. Proglucagon mRNA levels were then assessed in the absence or presence of the semi-synthetic cholic acid derivative and TGR5-specific agonist, 6 $\alpha$-ethyl-23(S)-methyl-cholic acid, referred to as INT-777 ${ }^{11,33}$. Interestingly, INT-777 robustly induced proglucagon mRNA levels in both cell lines, whereas no induction was observed in cells in which endogenous TGR5 expression was repressed (Fig. $2 \mathrm{c}$ and 2d). TGR5 activation is known to induce cAMP signaling and the proglucagon gene is an established target of cAMP signaling ${ }^{34-37}$. To obtain more mechanistic insight into the TGR5-mediated induction of proglucagon mRNA, we measured the activity of the proglucagon promoter by transfecting STC-1 and GLUTag cells with a luciferase reporter driven by the $2.3 \mathrm{~kb}$ upstream regulatory region of the proglucagon gene, which contains several cAMP responsive elements $^{34-37}$. In line with the TGR5-dependent increase of colonic proglucagon gene expression after AERs (Fig. 2b), INT-777 significantly induced proglucagon promoter activity in STC-1 and GLUTag cells. This effect was significantly blunted upon shTGR5 transfection, indicating that TGR5 is critical in mediating the increase of proglucagon promoter activity in response to INT-777 (Fig. 2e and $2 \mathrm{f}$ ).

Acute Colestilan treatment enhances GLP-1 release in a TGR5dependent manner. In addition to the known cholesterol- and glucose-lowering effects, AERs also curb body weight gain ${ }^{29,30,38,39}$. Although the mechanism for body weight loss is not entirely understood, it is generally accepted that sequestration of BAs by AERs largely contributes to the observed caloric loss, as a result of impaired mixed micelle formation and lipid absorption in the upper part of the small intestine. In our study, two weeks of resin treatment was already sufficient to induce a substantial reduction in body weight as well as an increase in fecal lipid loss in both TGR5 ${ }^{-1-}$ and $\mathrm{TGR}^{+/+}$mice (Table 1). To assess body weight independent actions of AERs on GLP-1 secretion, we also investigated the effects of acute administration of Colestilan in TGR $5^{-1-}$ and TGR $5^{+/+}$mice fed a high fat diet during 12 weeks. Interestingly, we observed that three hours of Colestilan feeding was sufficient to double GLP-1 levels after a test meal in TGR $5^{+/+}$mice, while no such effect was observed in TGR5 ${ }^{-1-}$ animals (Fig. 3a and $3 \mathbf{b}$ ). These data indicate

\begin{tabular}{|c|c|c|c|c|}
\hline LDL-cholesterol (mM) & $0.42 \pm 0.02$ & $0.35 \pm 0.03^{a}$ & $0.39 \pm 0.05$ & $0.32 \pm 0.03$ \\
\hline HDL-cholesterol (mM) & $4.50 \pm 0.16$ & $4.43 \pm 0.21$ & $4.49 \pm 0.22$ & $4.22 \pm 0.19$ \\
\hline Fecal weight (mg/day/g BW) & $12.30 \pm 3.54$ & $33.08 \pm 3.23^{a}$ & $11.87 \pm 2.35$ & $30.39 \pm 4.23^{a}$ \\
\hline Glycerol derivatives $(\mu \mathrm{g} / \mathrm{mg})$ & $0.18 \pm 0.02$ & $0.58 \pm 0.09^{a}$ & $0.24 \pm 0.03$ & $0.60 \pm 0.09^{a}$ \\
\hline Total cholesterol $(\mu \mathrm{g} / \mathrm{mg})$ & $3.88 \pm 1.23$ & $4.23 \pm 0.91$ & $3.23 \pm 1.78$ & $3.98 \pm 1.23$ \\
\hline Free fatty acids $(\mu \mathrm{g} / \mathrm{mg})$ & $5.45 \pm 1.56$ & $10.34 \pm 2.34^{a}$ & $6.23 \pm 1.34$ & $11.19 \pm 3.29^{a}$ \\
\hline Phospholipids ( $\mu \mathrm{g} / \mathrm{mg})$ & $3.39 \pm 0.62$ & $7.98 \pm 1.92^{a}$ & $11.13 \pm 3.23^{b}$ & $9.29 \pm 0.43$ \\
\hline Total bile acids $(\mu \mathrm{mol} / \mathrm{g})$ & $4.09 \pm 1.10$ & $10.29 \pm 0.98^{a}$ & $2.22 \pm 0.34^{\mathrm{b}}$ & $6.87 \pm 1.23^{a, b}$ \\
\hline
\end{tabular}

Colestilan $2 \%$ was supplemented in the diet for 2 weeks after 12 weeks of high fat feeding. Values represent mean $\pm \mathrm{SE}$.

a Statistically significant as compared to corresponding control group, $\mathrm{p}<0.05$

aStatistically significant as compared to corresponding
'Statistically significant between genotypes, $p<0.05$. 
a

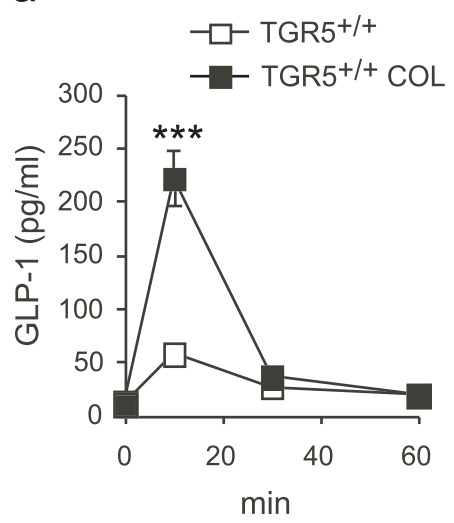

C

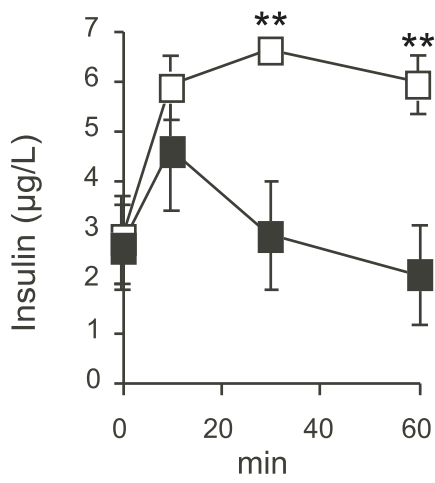

e

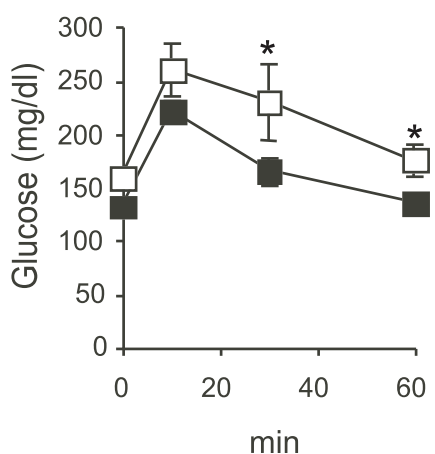

b

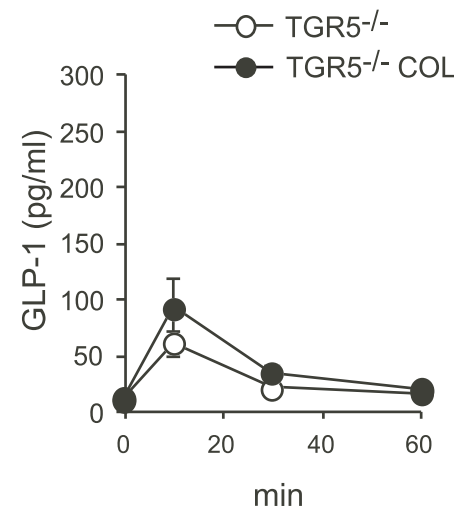

d

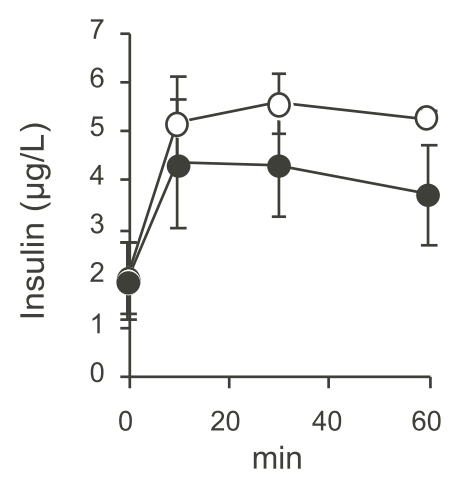

f

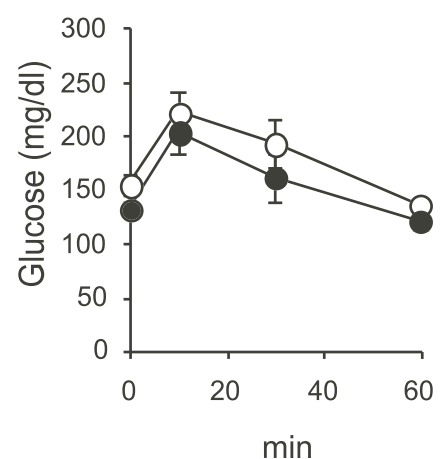

Figure 1 Potentiation of GLP-1 secretion by AERs is TGR5-dependent. (a-f) Analysis of plasma from diet-induced obese TGR5 ${ }^{+/+}$and TGR5 ${ }^{-/-}$mice after two weeks of treatment with Colestilan (COL; $\mathrm{n}=7$ per group). GLP-1 (a-b), insulin ( $\mathrm{c}-\mathrm{d}$ ), and glucose (e-f) levels at indicated time points after test meal. Data represent mean $\pm \mathrm{SE}$. ${ }^{*}$ Statistically significant, $\mathrm{p}<0.05, * * \mathrm{p}<0.01,{ }^{* * *} \mathrm{p}<0.001$.

that TGR5 is also critical in mediating the acute effects of AERs on GLP-1 secretion.

AERs potentiate GLP-1 release by delivering TGR5 agonists to the distal gut. Our observations indicate that long-term exposure of AERs triggers the induction of proglucagon gene expression in the distal gut. However it is unlikely that the previously observed transcriptional mechanism would entirely account for the rapid increase in circulating GLP-1 observed as shortly after resin treatment. We therefore hypothesized that AERs may also stimulate GLP-1 release by increasing the local availability of natural TGR5 agonists in the gut. To test this hypothesis, we first mapped the sites of GLP-1 release by monitoring GLP-1 secretion in several ex vivo cultured sections of the intestinal tract. Consistent with the enrichment of enteroendocrine Lcells in more distal gut segments ${ }^{40}$, ileal and colonic explants, but not duodenal explants, displayed the capacity to release GLP-1 (Fig. 4a).
Secretion of GLP-1 from colonic explants, however, was about 5-fold above that observed in ileal explants, indicating that colon is a major site of GLP-1 release in the intestinal tract (Fig. 4a). Exposure of colonic explants to either INT-777 or a mixture of natural bile acids, including litocholic acid (LCA) and deoxycholic acid (DCA) robustly potentiated GLP-1 release from explanted colon sections of $\mathrm{TGR}^{+/+}$, but not from TGR5 ${ }^{-/-}$mice (Fig. 4b), which is consistent with previous studies showing that activation of TGR5 in enteroendocrine cells by natural and semi-synthetic BAs induces the release of GLP- $1^{11,15,41}$. Interestingly, treating mice for two weeks with Colestilan also induced GLP-1 release in colon sections maintained ex vivo (Fig. 4c). This response was completely lost in the explants from TGR5 ${ }^{-I-}$ mice, further supporting the critical role of TGR5 in this process (Fig. 4c). To evaluate whether AER administration promotes the delivery of TGR5 agonists to the distal gut, we collected the colon content of untreated mice and mice treated for two weeks with 
a

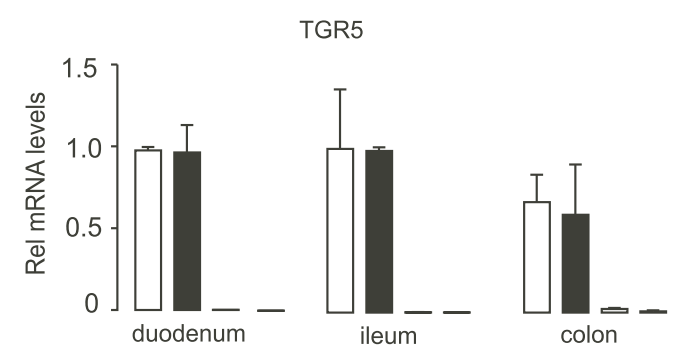

b

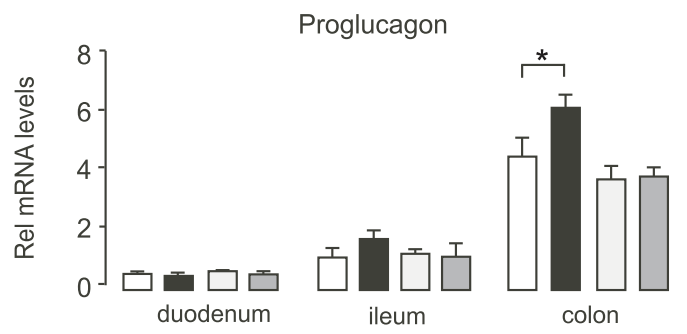

C

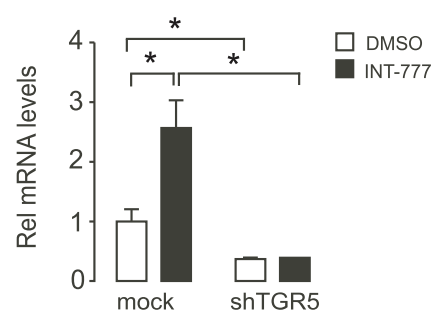

d

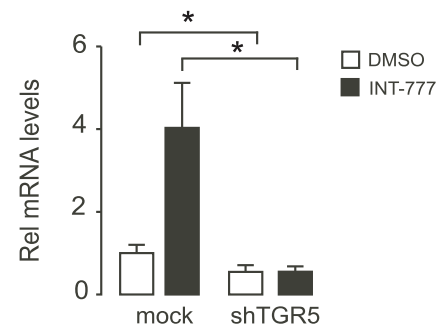

e

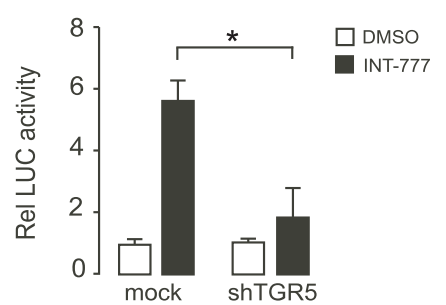

f

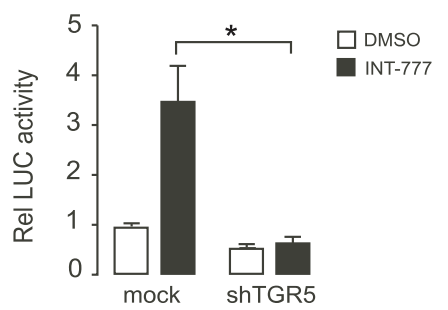

Figure 2 Enhanced proglucagon transcription in enteroendocrine cells in response to INT-777 requires TGR5. (a-b) TGR5 (a) and proglucagon (b) mRNA levels in the different intestinal sections of diet-induced obese TGR5 ${ }^{+/+}$and TGR5 ${ }^{-1-}$ mice after two weeks of treatment with Colestilan (COL; $\mathrm{n}=7$ per group). (c-d) Proglucagon mRNA levels in STC-1 (c) and GLUTag (d) mouse enteroendocrine cells transfected with shControl or shTGR5 vectors in the presence of $30 \mu \mathrm{M}$ INT-777 (black bars) or vehicle (white bars; $\mathrm{n}=3$ per group). (e-f) INT-777 induces proglucagon promoter activity via TGR5 in enteroendocrine cells. STC-1 (e) and GLUTag (f) mouse enteroendocrine cells were transfected with shControl or shTGR5 constructs in combination with the proglucagon luciferase reporter. Cells were then incubated with vehicle (white bars) or $30 \mu \mathrm{M}$ INT-777 (black bars; $\mathrm{n}=3$ per group) for 3 hours and assayed for luciferase activity. Data represent mean \pm SE. *Statistically significant, $\mathrm{p}<0.05$.

Colestilan and assessed their potency to activate TGR5 dependent cAMP response element (CRE) binding protein (CREBP) activation in $\mathrm{CHO}$ cells that were transfected with a TGR5 expression construct and a CRE driven luciferase reporter. Interestingly, while colon content from untreated mice only moderately increased luciferase activity in the presence of TGR5, a much more robust effect could be observed when cells were stimulated by the colon content from mice that were treated with Colestilan (Fig. 4d). The extent of activation by the colonic content of treated mice was also within the same range of activation by the TGR5 agonist, LCA, which served as a positive control in this experiment. We then evaluated whether TGR5 activation by the colon content from AER-treated mice is coupled to enhanced GLP-1 secretion using cultured colon segments. Consistent with our hypothesis, exposure of colon explants from $\mathrm{TGR} 5^{+/+}$mice with colon content from AER-treated wildtype mice significantly induced GLP-1 release from the colon explants (Fig. 4e). This potentiating effect was again significantly blunted when colon explants from $\mathrm{TGR}^{-1-}$ mice were exposed to colon content derived from AER-treated wildtype mice (Fig. 4e). No significant increase in GLP-1 secretion however was found upon exposure of control colon content to colon explants from both $\mathrm{TGR}^{+/+}$and $\mathrm{TGR} 5^{-/-}$mice. Altogether, these data provide evidence that colonic TGR5 is an essential component of AER-mediated stimulation of GLP-1 release from the intestinal L-cell.

\section{Discussion}

Several clinical studies reported the efficacy of AERs in the regulation of glycemic control ${ }^{24,42,43}$. In the current study, we provide evidence that the potentiating effects of AERs on GLP-1 secretion are critically dependent upon the G protein-coupled receptor TGR5. Our data indicate that AERs enhance delivery of natural TGR5 agonists to the colon and induce the expression of the GLP-1 precursor, preproglucagon, in a TGR5-dependent manner.
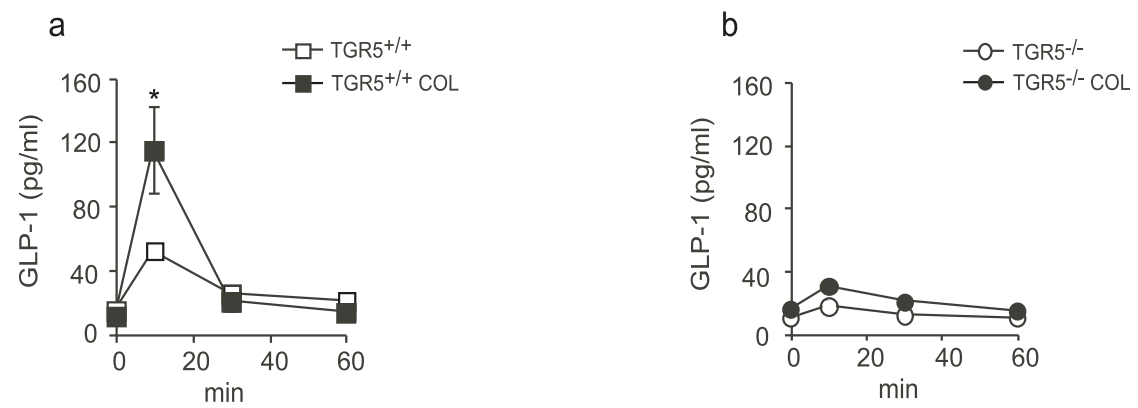

Figure 3 TGR5-dependent GLP-1 secretion is induced by acute exposure of AERs. (a-b) Plasma GLP-1 levels at indicated time points after test meal from high fat diet-induced obese $\mathrm{TGR}^{+/+}$mice (a) and TGR5 ${ }^{-1-}$ littermates (b) after three hours of treatment with Colestilan (COL; $\mathrm{n}=7 \mathrm{per}$ group). Data represent mean $\pm \mathrm{SE}$. *Statistically significant as compared to control group, $\mathrm{p}<0.05$. 
a

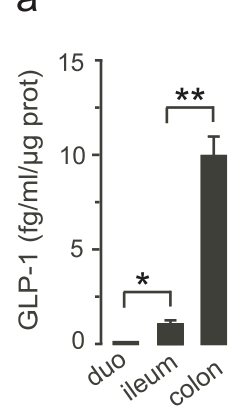

$\mathrm{b}$

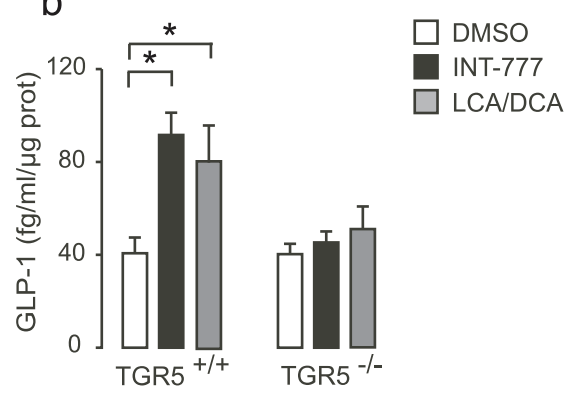

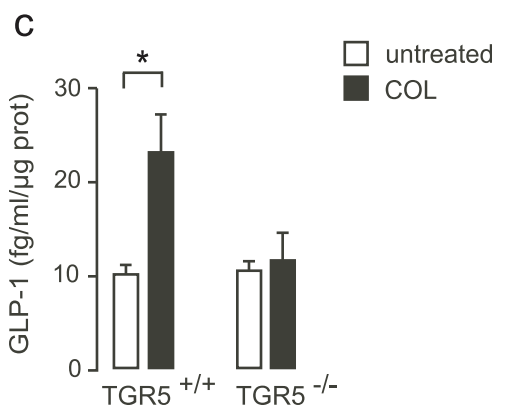

d

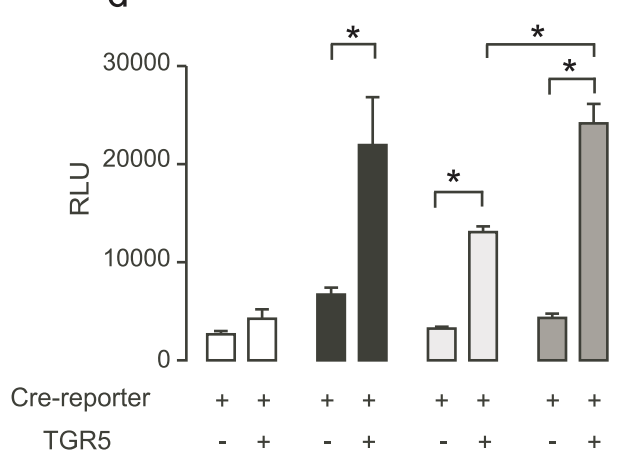

igure 4 TGR5 signaling is induced by colon content of mice treated with AERs. (a) GLP-1 release from explants of different intestinal segments of $\mathrm{TGR}^{+/+}$mice. Duodenum (duo). (b) GLP-1 release from colon explants of TGR5 ${ }^{+/+}$and TGR5 ${ }^{-1-}$ mice ( $\mathrm{n}=6$ per group) in response to 1 hour treatment with $30 \mu \mathrm{M}$ INT-777 or $15 \mu \mathrm{M}$ LCA/DCA. Mice were fed a high fat diet for 10 weeks prior to the collection of explants. (c) GLP-1 secretion from colon explants of TGR $5^{+/+}$and TGR5 ${ }^{-/-}$mice fed a high fat diet for 2 weeks and subsequently treated with Colestilan for two additional weeks ( $\mathrm{n}=6$ per group). (d) Luciferase activity in CHO cells transiently transfected with TGR5 expression vector and a cAMP response element (CRE)-driven luciferase reporter vector. Cells were treated with DMSO vehicle (white bars), $10 \mu \mathrm{M}$ litocholic acid (LCA; black bars), colon content collected from untreated wildtype mice (light gray bars), or colon content collected from Colestilan-treated wildtype mice (dark gray bars; $\mathrm{n}=5$ per group). The mice cohorts used for the collection of colon content were fed a high fat diet for 10 weeks prior treatment with Colestilan for 2 weeks. (e) GLP-1 release from colon explants of TGR $5^{+/+}$and TGR $5^{-1-}$ mice ( $\mathrm{n}=6$ per group) in response to 1 hour exposure of colon content derived from untreated wildtype mice (light gray bars), or colon content collected from Colestilan-treated wildtype mice $(n=5$ per group). Data represent mean \pm SE. *Statistically significant, $\mathrm{p}<0.05$; $* *: \mathrm{p}<0.01$.

In the past, multiple mechanisms have been proposed to explain the anti-diabetic effect of AERs. Most of these mechanisms invoked the BA sequestering properties of AERs and the resulting effects on BA signaling. In agreement with their role to interrupt the enterohepatic recirculation of BAs, AERs increase cholesterol and BA synthesis ${ }^{44}$. Despite the observation that resins render the BA pool more hydrophilic, no direct link between BA composition and glucose lowering has been demonstrated ${ }^{45}$. The glucose lowering effect of AERs has also been proposed to stem from changes in FXR activity in response to AERs ${ }^{46}$. Consistent with this hypothesis, AERs reduce blood levels of FGF15/19 $9^{32}$, which is an established FXR target in the intestine. Yet none of these hypotheses so far have been able to link AERs with enhanced GLP-1 release, an effect that is consistently observed after AER administration ${ }^{28,29,31}$.

In an attempt to understand the mechanistic basis of enhanced GLP-1 release in response to AERs, we have focused on the role of TGR5 in this process. Our hypothesis was based on previous findings from our laboratory indicating that BAs in the lumen of the intestine activate TGR5, stimulating the subsequent release of GLP-1. Unlike previous reports ${ }^{31}$, TGR5 mRNA expression was unchanged in response to Colestilan exposure, indicating that under our experimental conditions, AERs are not affecting TGR5 gene expression in enteroendocrine cells. In contrast, Colestilan increased proglucagon
mRNA levels in the colon in a TGR5-dependent manner, suggesting that resins enhance the transcription of the GLP-1 precursor gene in the enteroendocrine $\mathrm{L}$ cells of the distal gut. In agreement with this, TGR5 activation robustly induced the proglucagon promoter in enteroendocrine STC-1 and GLUTag cells, while this effect was significantly attenuated in cells in which TGR5 expression was silenced. The enhanced proglucagon promoter activity in response to TGR5 activation is in line with the fact that TGR5 induces cAMP signaling, which activates several cAMP responsive elements in the preproglucagon promoter ${ }^{34-37}$.

In this study, we did not observe significant changes in body weight between genotypes upon resin treatment. This could suggest that the body weight lowering action of TGR5 agonists is only effective when agonists can reach the systemic circulation. This would also be consistent with our previous studies showing that increased levels of plasma bile acids in high fat fed mice can induce energy expenditure and protect against diet-induced obesity ${ }^{10}$. In fact, AERs prevent bile acids from reaching the circulation while on the other hand they significantly concentrate bile acids in the intestinal lumen. At present, it is not unequivocally established whether basolateral or luminal TGR5 activation in enteroendocrine cells is important for GLP-1 secretion. Given the sequestering role of AERs, our current data would rather suggest an activation occurring at the luminal side of the enteroendocrine $\mathrm{L}$ cells, although we cannot exclude the 
possibility that small, but sufficient amounts of free bile acids can still reach the intestinal capillary system.

Most striking was the observation that Colestilan was able to rapidly induce GLP-1 release within three hours of administration. Interestingly, the colon content of AER-treated mice, but not that from untreated mice, enhanced GLP-1 release, suggesting that AERs may enrich the colon with TGR5 agonists. Given that TGR5 is a bile acid receptor, bile acids represent ideal candidates to fulfill this role, especially since the effect of AERs on GLP-1 release are strictly TGR5-dependent. It should be noted that bile acids are likely to activate TGR5 in their unbound form and that hence the ratio of bound: unbound bile acids in the colon following AER treatment may be critical. Furthermore, we postulate that the significant enrichment of other anionic biomolecules than BAs, such as phospholipids (Table 1) may ultimately increase the luminal ratio of free versus bound BAs in the distal gut area. This shift towards higher concentration of free BAs would in turn trigger TGR5 activity in the enteroendocrine L-cell of the colon, a hypothesis supported by our ex vivo data. Within this context, it is also noteworthy that TGR $5^{-1-}$ mice exhibit increased levels of fecal phospholipids already under basal conditions, suggesting that TGR5 is likely essential for normal phospholipid absorption and homeostasis.

Finally, the rapid and transient increase of GLP-1 in response to a test meal remains an observation that is still not completely understood. As only a small percent of a liquid load reaches the murine distal small intestine within 30 minutes, the finding that test mealstimulated GLP-1 release was increased 10 min after oral gavage in treated mice suggests that indirect neuro-endocrine mechanisms may also contribute. The $\mathrm{L}$-cell has been shown to be responsive to many cues triggered by the presence of nutrients in the proximal gut. Studies in rodents suggest that gastric inhibitory peptide (GIP) and gastrin-releasing peptide (GRP) could be mediators of this proximaldistal loop via activation of acetylcholine-dependent neuronal pathways ${ }^{47,48}$. In view of the expression of TGR5 in mesenteric neurons ${ }^{49}$, further studies with neuron-specific somatic TGR5 mutants may enable us to address this question.

In conclusion, our data provide evidence that the colon is a major source of enhanced GLP-1 secretion after administration of AERs. Resins increase GLP-1 secretion via activation of TGR5 and improve glycemic control in diet-induced models of obesity and insulin resistance. Moreover, we illustrate that AERs increase proglucagon gene promoter activity, resulting in increased proglucagon transcript levels. These findings may reveal an important mechanism by which AERs could exert its glucose-lowering effects in patients with type 2 diabetes.

\section{Methods}

Animal studies. Animal studies were performed according to regulations issued by the Swiss government and approved by the ethic veterinary committee of the canton of Vaud - Switzerland (Permit ID 2250). Animals were housed and bred according to standardized procedures ${ }^{50}$. Age-matched male mice were used for all experiments. TGR5 genetically engineered mouse models were described earlier. Eight week-old $\mathrm{TGR}^{+/+}$and TGR5 ${ }^{-/-}$mice were fed a HFD (60\% cal/fat, D12492; Research Diets) for 12 weeks or less as specified. Colestilan was admixed to the high fed diet at $2 \%$ (w/ w) and was given ad libitum to the mice during 2 weeks. Mice were sacrificed after 4 hours of fasting. For short-term Colestilan treatment, mice were initially fed a high fat diet for 12 weeks, fasted for 4 hours from 3:00 pm to 7:00 pm and refed with high fat diet pellets admixed with $2 \%(\mathrm{w} / \mathrm{w})$ Colestilan during 3 hours prior to the GLP-1 secretion assay.

For the in vivo plasma GLP-1 measurements, mice were gavaged with the dipeptidyl-peptidase (DPP)-IV inhibitor sitagliptin $(3 \mathrm{mg} / \mathrm{kg}) 60 \mathrm{~min}$ prior to the gavage of Ensure Plus at a dose of $10 \mathrm{ml} / \mathrm{kg}(1.5 \mathrm{Cal} / \mathrm{ml}$; Proteins: $15 \%$ of total Cal, Carbohydrates: $57 \%$ of total Cal, Fat: $28 \%$ of total Cal; Abott). Blood was collected $(100 \mu \mathrm{L})$ from the tail vein at the indicated times, transferred to EDTA tubes containing sitagliptin (Millipore), and kept on ice. Plasma was collected by centrifugation and transferred to ELISA plates to assess total GLP-1.

Ex vivo explant studies. For the intestinal explant study, diet induced obese mice were fed a high fat diet containing $2 \%$ Colestilan for two weeks. Animals were sacrificed and intestinal segments of $0.5 \mathrm{~cm}$ were dissected, cut into 5 smaller pieces, washed in PBS and maintained in DMEM 10\% FCS containing DPP-IV inhibitor.

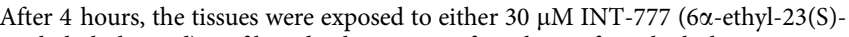
methyl-cholic acid), or filtered colon content for 1 hour after which the supernatant was frozen until total GLP-1 measurements were performed. Colon content was obtained from 5 Colestilan-treated and 5 control-treated mice, which had been on a high fat diet for 2 weeks. Colon content was pooled, resuspended in $3 \mathrm{ml}$ of HBSS medium (Sigma) and centrifuged at $4500 \mathrm{rpm}$ for $15 \mathrm{~min}$, after which the supernatant was filtered through a $0.22 \mu \mathrm{m}$ filter. The filtered solutions were diluted 1:10 and used in the TGR5 activation assay or used to treat colon explants as described in the figure legends.

GLP-1 secretion. Total GLP-1 measurements were performed according to the manufacturer's instructions (Total GLP-1 (7-36) amide; K150JVC-1 Mesoscale Discovery, Gaithersburg). For all GLP-1 assays, except for plasma measurements, concentrations of GLP-1 were normalized against total protein amount.

Insulin and glucose measurements. Glucose was assessed with a glucometer (Accucheck, Roche). Insulin was measured by ELISA according to the manufacturer's instructions (Mercodia Uppsala, Sweden)

Transient Transfections and Reporter Assays. Cells were transiently transfected with JetPei (Polyplus Transfection) according to the manufacturer's protocol. CHO cells were transiently transfected with TGR5 expression vector and a CRE-driven luciferase reporter ${ }^{51}$. After 24 hours of incubation, medium was replaced by serum free medium and cells were treated for 1 hour with the indicated compounds or with the colon content from untreated or Colestilan-treated mice. Cells were then lysed in Bright Glo reagent (Promega) and luciferase activity was determined as previously described $^{11}$. STC-1 and GLUTag enteroendocrine cells were transfected with a $2.3 \mathrm{~kb}$ rat proglucagon luciferase reporter ${ }^{52}$ in the presence of either a shControl or shTGR5 vector as previously described ${ }^{14}$. After 24 hours of incubation, medium was replaced by serum free medium and cells were treated for 4 hours with either vehicle or $30 \mu \mathrm{M}$ INT-777. Cells were then lysed in Bright Glo reagent (Promega) and luciferase activity was determined as previously described ${ }^{11}$. Luciferase activity was normalized against $B$-galactosidase levels. Experiments were performed three times, and data represent average values of quadruplicates of a representative experiment.

Quantitative RT-PCR. RNA was isolated from cells using TRIreagent (Ambion), after which cDNA was synthesized (Qiagen). Quantitative RT-PCR was performed using SYBR green (Roche) in the Lightcycler 480 II (Roche). All mRNA expression levels were corrected for expression of the housekeeping gene $36 \mathrm{~B} 4$ or $\beta 2$ microglobulin. Used primer sequences are available upon request.

Fecal lipid analysis. Fecal lipids were extracted essentially by using the method of $\mathrm{Folch}^{53}$, and were quantified using enzymatic assays. Briefly, feces were collected from mice individually housed over a 24 -h period. Aliquots of feces $(100 \mathrm{mg})$ were dried for $1 \mathrm{~h}$ at $70^{\circ} \mathrm{C}$, incubated with $2 \mathrm{ml}$ of chloroform-methanol $(2: 1)$ for $30 \mathrm{~min}$ at $60^{\circ} \mathrm{C}$ with constant agitation, and then centrifuged. Water $(1 \mathrm{ml})$ was added to the supernatant, and following vortexing, phase separation was induced by low-speed centrifugation (2,000 rpm for $10 \mathrm{~min}$ ). The lower chloroform phase was then removed and transferred to a new tube, and the sample was evaporated to dryness. Samples were then resuspended in $500 \mu \mathrm{l}$ chloroform-1\% Triton X-100, evaporated to dryness, and finally resuspended in $500 \mu \mathrm{l}$ of water, so that the final solvent was $1 \%$ Triton X-100 in water. The amount of total and free cholesterol (Wako, Neuss, Germany), triglycerides (BioMerieux, Marcy l'Etoile, France), free fatty acids (Wako), and phospholipids (Wako) in the fecal lipid extracts were then assayed using enzymatic kits according to manufacturers' protocols.

Fecal bile acids. Extraction and measurement of fecal bile acids were performed according to the method described by Dvir et al. ${ }^{54}$. Briefly, bile acids were extracted from dried feces $(100 \mathrm{mg})$ with $10 \mathrm{ml}$ of chloroform-methanol $(2: 1, \mathrm{v} / \mathrm{v})$ by agitation on a shaker table overnight. Two $\mathrm{ml}$ of $\mathrm{KCl}(3.7 \mathrm{~g} / \mathrm{L})$ were added and samples were centrifuged for $10 \mathrm{~min}\left(1,500 \mathrm{~g}, 4^{\circ} \mathrm{C}\right)$. The supernatant was removed, evaporated to dryness and dissolved in $1 \mathrm{ml}$ of methanol-water $(1: 1, \mathrm{v} / \mathrm{v})$. Bile acids were measured at $340 \mathrm{~nm}$ by colorimetry.

Statistical analysis. Data represent the means \pm standard errors of the means. Statistical analysis was performed using student's t-test. In case of multiple testing, the ANOVA test was used, followed by the student $t$ test. A $\mathrm{P}<0.05$ was considered statistically significant.

1. Thomas, C., Pellicciari, R., Pruzanski, M., Auwerx, J. \& Schoonjans, K. Targeting bile-acid signalling for metabolic diseases. Nat Rev Drug Discov 7, 678-693 (2008).

2. Trauner, M., Claudel, T., Fickert, P., Moustafa, T. \& Wagner, M. Bile acids as regulators of hepatic lipid and glucose metabolism. Dig Dis 28, 220-224 (2010).

3. Hylemon, P. B. et al. Bile acids as regulatory molecules. J Lipid Res 50, 1509-1520 (2009).

4. Keitel, V., Kubitz, R. \& Haussinger, D. Endocrine and paracrine role of bile acids. World J Gastroenterol 14, 5620-5629 (2008).

5. Kawamata, Y. et al. A G protein-coupled receptor responsive to bile acids. J Biol Chem 278, 9435-9440 (2003).

6. Maruyama, T. et al. Identification of membrane-type receptor for bile acids (MBAR). Biochem Biophys Res Commun 298, 714-719 (2002). 
7. Li, T. et al. The G protein-coupled bile acid receptor, TGR5, stimulates gallbladder filling. Mol Endocrinol 25, 1066-1071 (2011).

8. Maruyama, T. et al. Targeted disruption of $\mathrm{G}$ protein-coupled bile acid receptor 1 (Gpbar1/M-Bar) in mice. J Endocrinol 191, 197-205 (2006).

9. Keitel, V. et al. The membrane-bound bile acid receptor TGR5 is localized in the epithelium of human gallbladders. Hepatology 50, 861-870 (2009).

10. Watanabe, M. et al. Bile acids induce energy expenditure by promoting intracellular thyroid hormone activation. Nature 439, 484-489 (2006).

11. Thomas, C. et al. TGR5-mediated bile acid sensing controls glucose homeostasis. Cell Metab 10, 167-177 (2009).

12. Keitel, V., Donner, M., Winandy, S., Kubitz, R. \& Haussinger, D. Expression and function of the bile acid receptor TGR5 in Kupffer cells. Biochem Biophys Res Commun 372, 78-84 (2008).

13. Wang, Y. D., Chen, W. D., Yu, D., Forman, B. M. \& Huang, W. The G-Proteincoupled bile acid receptor, Gpbarl (TGR5), negatively regulates hepatic inflammatory response through antagonizing nuclear factor kappa light-chain enhancer of activated B cells (NF-kappaB) in mice. Hepatology 54, 1421-1432 (2011)

14. Pols, T. W. et al. TGR5 activation inhibits atherosclerosis by reducing macrophage inflammation and lipid loading. Cell Metab 14, 747-757 (2011).

15. Katsuma, S., Hirasawa, A. \& Tsujimoto, G. Bile acids promote glucagon-like peptide-1 secretion through TGR5 in a murine enteroendocrine cell line STC-1. Biochem Biophys Res Commun 329, 386-390 (2005).

16. Holst, J. J. The physiology of glucagon-like peptide 1. Physiol Rev 87, 1409-1439 (2007)

17. Nauck, M. A. et al. Glucagon-like peptide 1 inhibition of gastric emptying outweighs its insulinotropic effects in healthy humans. Am J Physiol 273, E981988 (1997).

18. Little, T. J. et al. Effects of intravenous glucagon-like peptide-1 on gastric emptying and intragastric distribution in healthy subjects: relationships with postprandial glycemic and insulinemic responses. J Clin Endocrinol Metab 91, 1916-1923 (2006).

19. Tang-Christensen, M., Vrang, N. \& Larsen, P. J. Glucagon-like peptide 1(7-36) amide's central inhibition of feeding and peripheral inhibition of drinking are abolished by neonatal monosodium glutamate treatment. Diabetes $47,530-537$ (1998).

20. Chelikani, P. K., Haver, A. C. \& Reidelberger, R. D. Intravenous infusion of glucagon-like peptide-1 potently inhibits food intake, sham feeding, and gastric emptying in rats. Am J Physiol Regul Integr Comp Physiol 288, R1695-1706 (2005)

21. Jones, M. R. et al. Rationale and design of a clinical trial to evaluate metformin and colesevelam $\mathrm{HCl}$ as first-line therapy in type 2 diabetes and colesevelam $\mathrm{HCl}$ in prediabetes. Curr Med Res Opin 25, 2239-2249 (2009).

22. Fonseca, V. A., Rosenstock, J., Wang, A. C., Truitt, K. E. \& Jones, M. R. Colesevelam $\mathrm{HCl}$ improves glycemic control and reduces LDL cholesterol in patients with inadequately controlled type 2 diabetes on sulfonylurea-based therapy. Diabetes Care 31, 1479-1484 (2008)

23. Bays, H. E. \& Cohen, D. E. Rationale and design of a prospective clinical trial program to evaluate the glucose-lowering effects of colesevelam $\mathrm{HCl}$ in patients with type 2 diabetes mellitus. Curr Med Res Opin 23, 1673-1684 (2007).

24. Davidson, M. H. Interrupting bile-acid handling and lipid and glucose control: Effects of colesevelam on glucose levels. J Clin Lipidol 2, S29-33 (2008).

25. Zieve, F. J., Kalin, M. F., Schwartz, S. L., Jones, M. R. \& Bailey, W. L. Results of the glucose-lowering effect of WelChol study (GLOWS): a randomized, double-blind, placebo-controlled pilot study evaluating the effect of colesevelam hydrochloride on glycemic control in subjects with type 2 diabetes. Clin Ther 29, 74-83 (2007).

26. Rigby, S. P. et al. Effects of colesevelam, rosiglitazone, or sitagliptin on glycemic control and lipid profile in patients with type 2 diabetes mellitus inadequately controlled by metformin monotherapy. Endocr Pract 16, 53-63 (2010).

27. Chen, L. et al. Inhibition of apical sodium-dependent bile acid transporter as a novel treatment for diabetes. Am J Physiol Endocrinol Metab 302, E68-76 (2012).

28. Shang, Q., Saumoy, M., Holst, J. J., Salen, G. \& Xu, G. Colesevelam improves insulin resistance in a diet-induced obesity (F-DIO) rat model by increasing the release of GLP-1. Am J Physiol Gastrointest Liver Physiol 298, G419-424 (2010).

29. Kobayashi, M. et al. Prevention and treatment of obesity, insulin resistance, and diabetes by bile acid-binding resin. Diabetes 56, 239-247 (2007).

30. Homma, Y. et al. Specific reduction of plasma large, light low-density lipoprotein by a bile acid sequestering resin, cholebine (MCI-196) in type II hyperlipoproteinemia. Atherosclerosis 129, 241-248 (1997).

31. Chen, L. et al. Cholestyramine reverses hyperglycemia and enhances glucosestimulated glucagon-like peptide 1 release in Zucker diabetic fatty rats. J Pharmacol Exp Ther 334, 164-170 (2010).

32. Beysen, C. et al. Effect of bile acid sequestrants on glucose metabolism, hepatic de novo lipogenesis, and cholesterol and bile acid kinetics in type 2 diabetes: a randomised controlled study. Diabetologia 55, 432-442 (2012).

33. Pellicciari, R. et al. Discovery of 6alpha-ethyl-23(S)-methylcholic acid (S-EMCA, INT-777) as a potent and selective agonist for the TGR5 receptor, a novel target for diabesity. J Med Chem 52, 7958-7961 (2009).

34. Gajic, D. \& Drucker, D. J. Multiple cis-acting domains mediate basal and adenosine $3^{\prime}, 5^{\prime}$-monophosphate-dependent glucagon gene transcription in a mouse neuroendocrine cell line. Endocrinology 132, 1055-1062 (1993).
35. Lu, F., Jin, T. \& Drucker, D. J. Proglucagon gene expression is induced by gastrinreleasing peptide in a mouse enteroendocrine cell line. Endocrinology 137, 37103716 (1996).

36. Gevrey, J. C. et al. Protein hydrolysates stimulate proglucagon gene transcription in intestinal endocrine cells via two elements related to cyclic AMP response element. Diabetologia 47, 926-936 (2004).

37. Drucker, D. J. \& Brubaker, P. L. Proglucagon gene expression is regulated by a cyclic AMP-dependent pathway in rat intestine. Proc Natl Acad Sci U S A 86, 3953-3957 (1989).

38. Meneghini, L. F. et al. Weight beneficial treatments for type 2 diabetes. J Clin Endocrinol Metab 96, 3337-3353 (2011).

39. Koike, K., Murakami, K., Nozaki, N., Sugiura, K. \& Inoue, M. Colestilan, a new bile acid-sequestering resin, reduces bodyweight in postmenopausal women who have dieted unsuccessfully. Drugs R D 6, 273-279 (2005).

40. Eissele, R. et al. Glucagon-like peptide- 1 cells in the gastrointestinal tract and pancreas of rat, pig and man. Eur J Clin Invest 22, 283-291 (1992).

41. Parker, H. E. et al. Molecular mechanisms underlying bile acid-stimulated glucagon-like peptide-1 secretion. Br J Pharmacol 165, 414-423 (2012).

42. Goldfine, A. B. Modulating LDL cholesterol and glucose in patients with type 2 diabetes mellitus: targeting the bile acid pathway. Curr Opin Cardiol 23, 502-511 (2008).

43. Hofmann, A. F. Bile acid sequestrants improve glycemic control in type 2 diabetes: a proposed mechanism implicating glucagon-like peptide 1 release. Hepatology 53, 1784 (2011)

44. Einarsson, K. et al. Bile acid sequestrants: mechanisms of action on bile acid and cholesterol metabolism. Eur J Clin Pharmacol 40 Suppl 1, S53-58 (1991).

45. Herrema, H. et al. Bile salt sequestration induces hepatic de novo lipogenesis through farnesoid X receptor- and liver X receptor alpha-controlled metabolic pathways in mice. Hepatology 51, 806-816 (2010).

46. Prawitt, J., Caron, S. \& Staels, B. Bile acid metabolism and the pathogenesis of type 2 diabetes. Curr Diab Rep 11, 160-166 (2011).

47. Rocca, A. S. \& Brubaker, P. L. Role of the vagus nerve in mediating proximal nutrient-induced glucagon-like peptide-1 secretion. Endocrinology 140, 16871694 (1999).

48. Roberge, J. N., Gronau, K. A. \& Brubaker, P. L. Gastrin-releasing peptide is a novel mediator of proximal nutrient-induced proglucagon-derived peptide secretion from the distal gut. Endocrinology 137, 2383-2388 (1996).

49. Poole, D. P. et al. Expression and function of the bile acid receptor GpBAR1 (TGR5) in the murine enteric nervous system. Neurogastroenterol Motil 22, 814825, e227-818 (2010).

50. Argmann, C. A. \& Auwerx, J. Minimizing variation due to genotype and environment. Curr Protoc Mol Biol Chapter 29, Unit 29A 22 (2006).

51. Sato, H. et al. Novel potent and selective bile acid derivatives as TGR5 agonists: biological screening, structure-activity relationships, and molecular modeling studies. J Med Chem 51, 1831-1841 (2008).

52. Gauthier, B. R. et al. Hepatic nuclear factor-3 (HNF-3 or Foxa2) regulates glucagon gene transcription by binding to the G1 and G2 promoter elements. Mol Endocrinol 16, 170-183 (2002).

53. Folch, J., Lees, M. \& Sloane Stanley, G. H. A simple method for the isolation and purification of total lipides from animal tissues. J Biol Chem 226, 497-509 (1957).

54. Dvir, I. et al. Soluble polysaccharide and biomass of red microalga Porphyridium sp. alter intestinal morphology and reduce serum cholesterol in rats. Br J Nutr 84, 469-476 (2000)

\section{Acknowledgments}

Colestilan and INT-777 were a gift of Mitsubishi Pharmaceuticals and Intercept Pharmaceuticals. We thank Drs Daniel Drucker and Fiona Gribble for the GLUTag cells, and Dr. Jacques Philippe for the proglucagon luciferase reporter. This research was supported by the Ecole Polytechnique Fédérale de Lausanne, Swiss National Science Foundation (31003A_125487), and Nestlé. T.W.H.P. and M.N. are supported by FEBS and AXA fellowships. J.A. is Nestlé Chair in Energy Metabolism and A.M. is supported by a fellowship from the Canadian Institutes of Health Research.

\section{Author contributions}

T.H. and T.W.H.P contributed equally to this work. T.H., T.W.H.P and A.M. wrote the paper and contributed to all experiments. M.W. and M.N. contributed to the experiments. J.A. and K.S. supervised the project and wrote the paper.

\section{Additional information}

Competing financial interests: The authors declare no competing financial interests.

License: This work is licensed under a Creative Commons

Attribution-NonCommercial-NoDerivative Works 3.0 Unported License. To view a copy of this license, visit http://creativecommons.org/licenses/by-nc-nd/3.0/

How to cite this article: Harach, T. et al. TGR5 potentiates GLP-1 secretion in response to anionic exchange resins. Sci. Rep. 2, 430; DOI:10.1038/srep00430 (2012). 